\title{
Nachricht von Radio Eriwan, Teil 1
}

\section{Bericht von einer Gastprofessur in Armenien}

\section{Die Kooperation von ÖGKJ, AAF und $\mathrm{OMI}$}

Seit mittlerweile mehr als 15 Jahren besteht eine enge Zusammenarbeit zwischen der Österreichischen Gesellschaft für Kinder- und Jugendheilkunde (ÖGKJ) und der American Austrian Foundation (AAF) bzw. dem Open Medical Institute (OMI), welches in mehreren Ländern Vertretungen hat. Im Rahmen dieser Kooperation werden neben den Salzburg Seminars immer wieder Satellitensymposien v. a. in „östlichen Ländern" veranstaltet, zu denen österreichische Kolleginnen und Kollegen zu einer Gastprofessur eingeladen werden. Eine solche durften wir, Univ.-Prof. Dr. Almuthe Hauer und ich, im Oktober 2016 in Armenien wahrnehmen.

\section{Professorship in Jerewan/Eriwan}

Als mehrfach bei den Salzburg Seminars als Vortragende und Co-CourseDirectors Tätige wurden wir im Frühjahr 2016 mit dem Ersuchen konfrontiert, in Eriwan ein zweitägiges Satellitensymposium abzuhalten. In weiterer Folge wurden mit der armenischen Kollegin Dr. Gayane AMARYAN und dem OMI Armenia Datum, Tagungsort und Inhalte des Symposiums festgelegt. Wir wurden jeweils zu drei Hauptvorträgen eingeladen (• Tab. 1), diese wurden ergänzt durch einige ausgewählte Kasuistiken armenischer Kolleginnen und Kollegen [1].

Der Vortragssaal am Arabkir Medical Center (Institute of Child and Adolescent Health) war schließlich mit ca. 150 Teilnehmerinnen und Teilnehmern sehr gut gefüllt. Diese zeigten großes Interesse an den präsentierten Inhalten, dies resultierte in interessierten Fragen und einer angeregten Diskussion.

Im Rahmen der Tagung hatten wir auch Gelegenheit, führende armeni-

$\begin{array}{ll}\text { Tab. } 1 & \\ \text { A. Hauer } & \text { R. Kerbl } \\ \text { - Acute and chronic diarrhea } & - \text { Lymphadenopathy in children } \\ \text { - Malabsorption and failure to thrive } & - \text { Fever of unknown origin (FUO) } \\ \text { - Relevant food intolerance } & - \text { Sudden infant death syndrome (SIDS) }\end{array}$

sche Pädiater zu treffen und unsere Erfahrungen auszutauschen. So hatten wir ein "Science Meeting“ beim Klinikvorstand Prof. Ara Babloyan, der auch auf eine Karriere als Gesundheitsminister zurückblicken kann, sich aber dann doch wieder auf seine Tätigkeit als Kliniker zurückgezogen fühlte. Ein Bild von Charles Aznavour in Professor Babloyans Büro erinnert daran, dass dieser berühmte Sänger aus Armenien stammt und auch pädiatrische Projekte dort großzügig unterstützt hat.

Im Rahmen des Meetings lernten wir auch Professor Sergey Sargsyan kennen - er ist Generalsekretär der Armenischen Gesellschaft für Pädiatrie und hat zuletzt im Rahmen eines EPA-Projektes im Journal of Pediatrics über das armenische Gesundheitssystem berichtet [2], so wie es der Verfasser dieser Zeilen für Österreich tun durfte [3].

\section{Univ.-Prof. Dr. Reinhold Kerbl}

\section{Literatur}

1. http://www.aaf-online.org/tl_files/externals/downloads/satellites/docs/2016/ Schedule_Armenia_Pediatrics.pdf

2. Sargsyan S, Movsesyan Y, Melkumova M, Babloyan A (2016) Child and Adolescent Health in Armenia: Experiences and Learned Lessons. J Pediatr 177S:S21-S34

3. Kerbl R, Ziniel G, Winkler P, Habl C, Püspök R, Waldhauser F (2016) Child Health Care Services in Austria. J Pediatr 177S:S35-S47

Paediatr. Paedolog. Austria 2018 · 53:41 https://doi.org/10.1007/s00608-0170536-3 (c) Springer-Verlag GmbH Austria, ein Teil von Springer Nature 2017

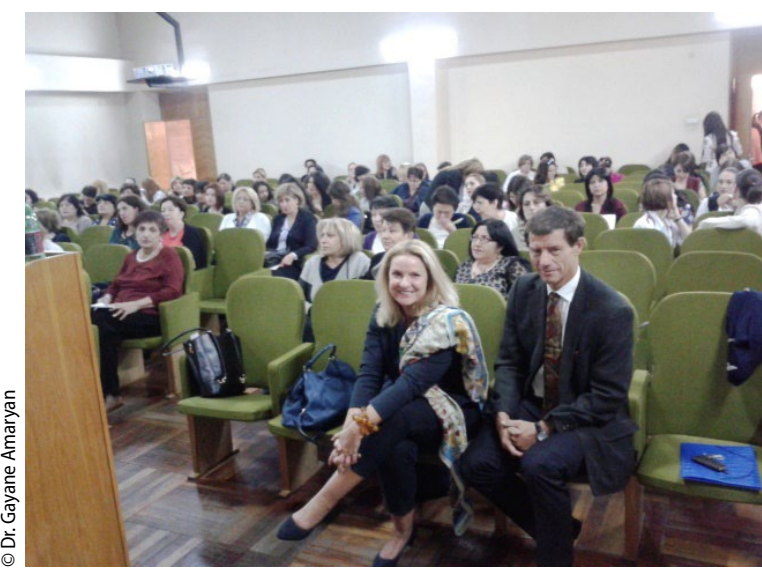

$\Delta$ Almuthe Hauer und Reinhold Kerbl bei ihrer Gastprofessur in Eriwan

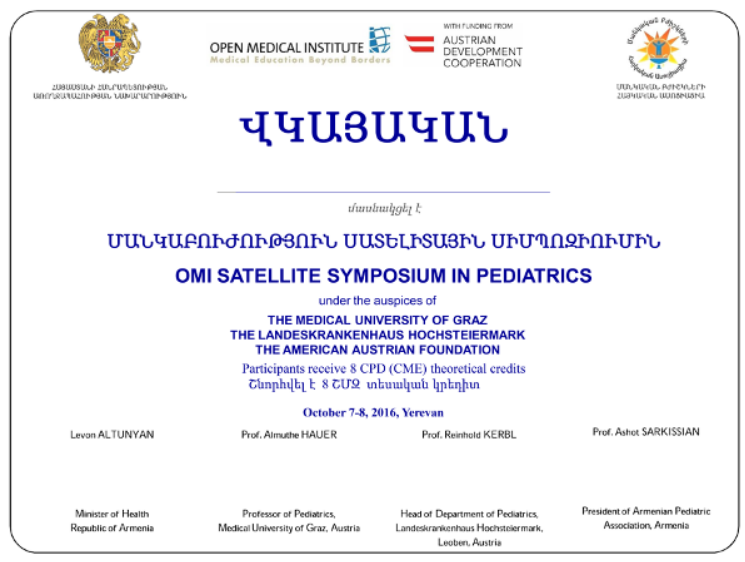

$\Delta$ Diplom des Satellitensymposiums in Eriwan/Armenien 